\title{
Toughening Analysis of Eutectic Ceramic Composite with Damage
}

\author{
Liu Heng ${ }^{1, a}$, Zhao Gang ${ }^{1, a}$, RunZe Xiong ${ }^{1, a}$ \\ ${ }^{1} \mathrm{NO} 63981$ unit of PLA, Wu Han, China \\ a18523397490@163.com
}

Keywords: Damage rod-shaped eutectic; critical fracture stress; bridging toughening mechanism; pull-out toughening mechanism

\begin{abstract}
The damage variables were defined by the microstructure of rod-shaped eutectic with parallel nano-micron fibers. The maximum strain criterion was used for determining the loading function. According to the attenuation characteristic of eutectic rigidity, the critical fracture stress of the damage rod-shaped eutectic was obtained by damage variable maximizing. On the basis of the work done by frictional force over the pull-out length, bridging toughening mechanism and pull-out toughening mechanism of damage rod-shaped eutectics were constructed. The result shows that damage in the eutectic reduces the eutectic rigidity, thus fracture strength decreases and the eutectic contribution to composite ceramic bridging toughening and pull-out toughening decreases.
\end{abstract}

\section{Introduction}

With highest mechanical property at both room temperature and high temperature, the lowest high-temperature creep property and the highest room-temperature fracture toughness, directional solidification oxide eutectic composite ceramic is paid much attention to by researchers both at home and abroad. On the basis of strong confinement property of the phases in the rod-shaped eutectic, referonce [1] confirms the microscomic stress field of the rod-shaped eutectic through fiber and matrix interfacial shear stress. It is shown in the paper that stressed concentration caused by two phase interface dislocation pile-up makes the maximum stress within the eutectic so that the analytical expression about the theoretical fracture strength of the rod-shaped eutectic is obtained. On the basis of the theoretical fracture strength of the rod-shaped eutectic, referonce [2] analyzes bridging toughening mechanism and pull-out toughening mechanism of the rod-shaped eutectic and establishes bridging and the pull-out toughening model based on theoretical fracture strength of the eutectic. In these studies, the theoretical fracture strength of the eutectic is confirmed when the stress at the peril point reaches the fracture strength of the molecular structure theory. It has nothing to do with nano-micron fiber distribution within the eutectic. However, the fracture of the eutectic is gradually caused by the microscopic damage starting from the peril point, which has much to do with nano-micron fiber distribution within the eutectic.

\section{Critical fracture stress of damage eutectic}

There are a lot of rod-shaped eutectic within the eutectic composite ceramic, thus critical fracture stress of the rod-shaped eutectic has to be determined first before analyzing the ceramic composite damage rule. And rod-shaped eutectic can be regarded as a special inclusion composed by parallel fiber inclusions, eutectic interfaces as well as the matrix. As it is shown in referonce [3], the theoretical fracture strength of compound eutectic can be calculated as follows:

$$
\sigma_{f u}=\frac{2 E_{22}}{n\left(A E_{22}+E_{b} v_{21}\right) \tanh (n l / d)}\left(\tau_{i}+\sqrt{\frac{3 E_{0} \gamma_{0}}{2 d}}\right)
$$

In this type: $E_{22}$ refers to elasticity modulus along the vertical direction of the eutectic while perpendicular to the direction of fiber inclusions; $E_{0}$ refers to elasticity modulus of eutectic matrix ; $E_{b}$ refers to elasticity modulus of fiber inclusions within the eutectic; $\gamma_{0}$ refers to free surface 
energy of compound eutectic matrix; $\tau_{\mathrm{i}}$ refers to resistance in the lattice dislocation motion; $v_{21}=1-E_{22} / 2 \mu_{12}$ refers to transverse and longitudinal poisson ratio of the eutectic, $\mu_{12}$ refers to longitudinal shear modulus of the eutectic; $n^{2}=1 \frac{1}{2}, \Gamma=\mu_{12} / \mu, \mu$ refers to shear modulus of

$$
\overline{\left(1+v_{12}\right) \Gamma l n\left(\frac{\pi}{f_{b}}\right)}
$$

ceramiccomposite; $A=\frac{\Gamma}{2\left(1+\Gamma-2 v_{12}\right)}\left[\frac{(1+\Gamma)(1-v)\left(1+v_{12}\right)}{(1+v)}-\left(1+\Gamma+v_{12} \Gamma-3 v_{12}\right)\right], v$ refers to poisson ratio of ceramic composite ; $l$ and $d$ respectively refer to length and diameter of fiber inclusions within the eutectic; $E_{22}, \mu_{12}, \mu$ and $v$ can be calculated with four-phase-model method ${ }^{[6]}$.

In type (1), the theoretical fracture strength of the eutectic is determined when the stress at the peril point reaches the fracture strength of the molecular structure theory, having nothing to do with nano-micron fiber distribution within the eutectic.As a matter of fact, the fracture of the eutectic is gradually caused by the microscopic damage starting from the peril point. According to the analysis of the stress field, damage will first appear in the matrix around fiber after loading. At the same time, the eutectic rigidity will decay. Then the influence damage has on elastic property will be analyzed with damage variable.

According to the microstructure property of compound eutectic, four-phase-model method should be applied ${ }^{[6]}$, and the coordinate system in Fig.1 will be chosen. A standard orthogonal vector set is defined $\left(\boldsymbol{e}_{1}, \boldsymbol{e}_{2}, \boldsymbol{e}_{3}\right) \cdot \boldsymbol{e}_{1}$ refers to the unit vector parallel to the direction of fiber, then two orthogonal vectors $\boldsymbol{e}_{2}$ and $\boldsymbol{e}_{3}$ are chosen in the plane orthogonal to $\boldsymbol{e}_{1}$.

Suppose nano-micron fiber, interface phase and matrix are isotropic, and nano-micron fiber is distributed synclastically in parallel, then the eutectic is transverse isotropic and the shearing strain in the plane $\gamma_{23}$ will be zero. Meanwhile, suppose the density of the material is constant, then the total complementary free energy will be $\int_{V} \psi d V$, in which $\psi$ refers to complementary free energy in unit volume. It is defined as follows.

$$
\psi=\frac{\sigma_{11}^{2}}{2\left(1-d_{1}\right) E_{11}}+\frac{1}{2 E_{22}}\left(\frac{\sigma_{22}^{2}}{1-d_{2}}+\frac{\sigma_{33}^{2}}{1-d_{3}}\right)-\frac{v_{12}}{E_{11}}\left(\sigma_{22}+\sigma_{33}\right) \sigma_{11}-\frac{v_{23}}{E_{22}} \sigma_{22} \sigma_{33}+\frac{\sigma_{12}^{2}+\sigma_{13}^{2}}{2\left(1-d_{6}\right) G_{12}}
$$

In this type, $E_{11}$ refers to elasticity modulus that the eutectic is parallel to the direction of fiber inclusions ${ }^{[6]} ; \alpha_{11}$ and $\alpha_{22}$ respectively refer to thermal expansivity parallel and vertical to the direction of fiber inclusions ${ }^{[7]}, d_{1}, d_{2}, d_{3}$ and $d_{6}$ are damage variables defined by vector $\left(\boldsymbol{e}_{1}, \boldsymbol{e}_{2}, \boldsymbol{e}_{3}\right)$. Damage variable $\mathrm{d}_{1}$ refers to the influence damage has on vertical rigidity of the compound eutectic along the direction of fiber inclusions. Damage variables $d_{2}$ and $d_{3}$ refer to the influence damage has on transverse rigidity of the eutectic relative to the plane of fiber inclusions. And damage variable $d_{6}$ will have influence on the eutectic shear modulus.

Damage variables $d_{1}, d_{2}$ and $d_{3}$ depends on transverse and vertical stress, which are described as follows.

$$
d_{1}=d_{\mathrm{T}+}^{1} \frac{\left\langle\sigma_{11}\right\rangle}{\left|\sigma_{11}\right|}+d_{\mathrm{T}-}^{1} \frac{\left\langle-\sigma_{11}\right\rangle}{\left|\sigma_{11}\right|}, d_{2}=d_{\mathrm{T}+}^{2} \frac{\left\langle\sigma_{22}\right\rangle}{\left|\sigma_{22}\right|}+d_{\mathrm{T}-}^{2} \frac{\left\langle-\sigma_{22}\right\rangle}{\left|\sigma_{22}\right|}, d_{3}=d_{\mathrm{T}+}^{3} \frac{\left\langle\sigma_{33}\right\rangle}{\left|\sigma_{33}\right|}+d_{\mathrm{T}-}^{3} \frac{\left\langle-\sigma_{33}\right\rangle}{\left|\sigma_{33}\right|}
$$

In this type, according to McCauey operation, $\langle x\rangle$ can be defined as $\langle x\rangle=(x+|x|) / 2, d_{\mathrm{T}^{+}}$and $d_{\mathrm{T}_{-}}$are damage variables in directions when pulled and pressed.

According to referonce [1], due to microcosmic heterogeneous plastic flow, the matrix stress around the nano-micron fiber in the eutectic which is under loading should first reach the theoretical fracture strength. Thus the eutectic microscopic damage will start from the matrix surrounding the fiber. It is apparent that the microscopic damage of the matrix surrounding the fiber has little influence on the eutectic vertical damage variables along the direction of the fiber, which can be ignored.

When the eutectic has some compressed stress along the direction of $\boldsymbol{e}_{2}$, since the eutectic microscopic damage surrounding the fiber will not propagate, then $d_{\tau^{-}}^{2}=0$ will be an option. 
Meanwhile, vectors $\boldsymbol{e}_{2}$ and $\boldsymbol{e}_{3}$ are the only ones which will influence in the transverse isotropic plane, therefore, $\sigma_{22}$ and $\sigma_{33}$ should have the same effect, which means $d_{2}=d_{3}$.In the transverse isotropic plane, the same axis is shared by principal stress and principal strain. Then the corresponding shear modulus will be calculated with the following expression.

$$
\mu_{23}^{\prime}=\frac{E_{22}}{2\left[1+v_{23}\left(1-d_{2}\right)\right]}
$$

Suppose the nano-micron fiber within the eutectic is distributed in square, and square cylinder of a single fiber taken from the eutectic is a unit cell, as is shown in Fig. 2.

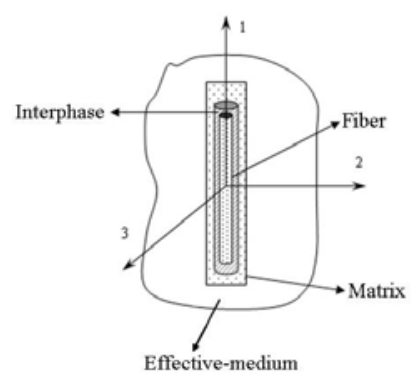

Figure 1. Compound eutectic four-phase-model

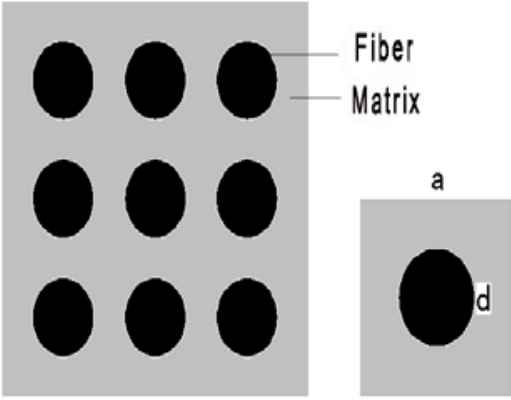

Figure 2. Permutation of fiber within the eutectic

As is shown in Fig.2, nano-micron fiber volume fraction $f_{b}$ is as follows.

$$
f_{b}=\frac{\pi d^{2} l}{4 a^{2} l}=\frac{\pi d^{2}}{4 a^{2}}
$$

Apparently, the maximum fracture area surrounding the fiber is the separation of the half cylinder domain. As for unit cell, the minimum zone of the composites can be calculated as $A_{\min }=(a-d) l$, and the damage variable has to be calculated as follows.

$$
d_{2 \max }=1-\frac{A_{\min }}{A}=1-\frac{(a-d) l}{a l}=\frac{d}{a}=\sqrt{\frac{4 f_{b}}{\pi}}
$$

Then the range of damage variable $d_{2}$ is as follows. $0 \leq d_{2} \leq \sqrt{\frac{4 f_{b}}{\pi}}$

If stress migration and consumption caused by shear load are ignored, then

$$
d_{6}=0
$$

Next,the damage eutectic flexibility tensor can be obtained.

Because the influence that damage has on the eutectic along the direction vertical to the fiber is so small after loading that it can be ignored, the value of $E_{11}$ will remain the same before and after the material damage. Since shear stress parameter will have no influence on the value of shear damage variable, and the effect that normal stress has on the crack surface and shear load has small influence on damage variable, the influence is ignored in the model. The influence of the damage vertical to the fiber is ignored, so when there is stress along the direction of $e_{2}$ in the eutectic, microscopic damage will not extend, in other words, along the direction of $\sigma_{22}, d_{L-}=0$. According to the analysis above, when damage appears in the eutectic loading, the transverse elasticity modulus of the material will decay. When damage variable $d_{2}$ reaches the maximum, the compound eutectic elasticity modulus will be as follows.

$$
E_{11}{ }^{\prime}=E_{11}, \quad E_{22}{ }^{\prime}=\left(1-\sqrt{\frac{4 f_{b}}{\pi}}\right) E_{22}
$$

Suppose the loading is along the direction vertical to the eutectic, when tensile stress $\sigma$ appears along the direction of $\sigma_{22}$ of the eutectic, $\sigma_{11}=\sigma_{33}=0, \sigma_{22}=\boldsymbol{\sigma}$.According to referonce [8], the strain along the axis of the eutectic in the matrix is the primary factor of local damage. According to the maximum strain theory, $\varepsilon_{\max }=\varepsilon_{2}$.

A loading function $\varphi$ is defined as follows. 


$$
\varphi=\frac{E_{c}}{X_{\mathrm{T}}}\left\langle\varepsilon_{c}\right\rangle
$$

In this type, $E_{\mathrm{c}}$ is the elasticity modulus along the direction of $\varepsilon_{\mathrm{c}}, X_{\mathrm{T}}=1-d_{2}$. Loading function $\varphi$ is determined by strain tensor,elasticity and strength properties. Because $\varepsilon_{22}=\frac{\sigma}{\left(1-d_{2}\right) \mathrm{E}_{22}}>0$, then

$$
\varphi=\frac{\sigma}{\left(1-d_{2}\right)^{2}}
$$

When no damage is in the compound eutectic, then $\varphi=\sigma_{f u}$. Then type(1) is substituted in the type above, when damage variable $d_{2}$ reaches maximum, the eutectic critical fracture stress can be obtained as follows.

$$
\sigma_{d u}=\frac{2 E_{22} \cdot}{n\left(A E_{22} S_{11}+E_{b} v_{21}\right) \tanh (n l / d)}\left(1-\sqrt{4 f_{b} / \pi}\right)^{2} \sqrt{\frac{3 E_{0} \gamma_{0}}{2 d}}
$$

As for ceramic composite, the resistance $\tau_{\mathrm{i}}$ that lattice brings to dislocation motion can be ignored, then $\tau_{i}=0$. Type(11) suggests that the compound eutectic fracture stress has much to do with the content, shape and size of fiber inclusions, elasticity modulus and free surface energy of the matrix as well as the fiber eutectic elastic constant.

\section{Bridging toughening and pull-out toughening of damage eutectic}

Eutectic ceramic composite is mainly constituted by compound eutectic containing parallel fiber inclusions, as well as few matrix particles and transformation particles surrounding the eutectic. Suppose there is a crack in ceramic composite, after loading, the eutectic on the surface of the crack keeps the crack from spreading, then the bridging stress of the eutectic on the surface of the crack is obtained as follows ${ }^{[2]}$.

$$
\sigma_{f}\left(L_{s}\right)=\frac{\sigma_{f}}{2}\left(\sqrt{1+\frac{16 E_{22}^{\prime} \tau u}{R \sigma_{f}^{2} \sin \alpha}}+1\right)
$$

In this type, $\sigma_{\mathrm{f}}$ refers to the eutectic stress in the far-field region far from the crack surface. $\mathrm{R}$ refers to the eutectic radius. $\tau$ refers to shear stress at the sliding part on the eutectic surface.u refers to crack opening displacement. $\alpha$ is the included angle of the bridging eutectic and the crack direction. When the eutectic on the surface of the crack keeps the crack from extending,an abhesion length will be generated for the eutectic. The eutectic has two kinds of toughening modes. The first one is that a force will be generated by the bridging eutectic to close the crack on the surface of the crack, which will diminish the stress concentration of the crack tip. The other is that additional work of fracture will also be generated due to the pull-out of abhesion eutectic. The toughening mechanism of this two modes will be analyzed in the following part.

Because the eutectic bridging force on the surface of the crack will bring crack closure, the stress concentration of the crack tip is diminished. When the direction of the crack extension differs from the eutectic vertical, the eutectic with crack bridging will release its own elastic strain energy to consume the mechanical energy necessary for the crack extension. Therefore, shielding effect will be generated for the crack tip to keep the crack from extending and to induce crack bridging toughening mechanism.

With the influence of the eutectic position and volume fraction, according to type(12), the bridging load that rod-shaped eutectic makes the crack close is as follows.

$$
T=f_{f} \sigma_{f}\left(L_{s}\right) \sin \alpha=\frac{f_{f} \sigma_{f} \sin \alpha}{2}\left(\sqrt{1+\frac{16 E_{22}^{\prime} \tau u}{R \sigma_{f}^{2} \sin \alpha}}+1\right)
$$


In this type, $f_{f}$ is the eutectic volume fraction. The energy dissipation in the bridging $\Delta J_{1}$ is the function of bridging load $T$ and opening displacement $u . \Delta J_{1}=\int_{0}^{u_{\max }} T d u$.

Suppose the rod-shaped eutectic is distributed three-dimensionally at random. According to referonce [2], it can be calculated as follows.

$$
\Delta J_{1}=\frac{\chi f_{f} R \sigma_{d u}^{3}}{E \tau}
$$

In this type, $\mathrm{E}$ is elasticity modulus of composite ceramic, $\chi$ is proportionality coefficient related to $E_{22}{ }^{\prime}$ and $E^{[2]}$. According to energy dissipation, the relation of fracture toughness and energy dissipation can be described as $\Delta K_{C}=(E \Delta J)^{1 / 2}$. Suppose the eutectic aspect ratio is $\lambda=L / 2 R$, then the eutectic bridging toughening value can be as follows.

$$
\Delta K_{C 1}=\sqrt{\frac{\chi f_{f} L \sigma_{d u}^{3}}{2 \lambda \tau}}
$$

It is suggested from the type above that the eutectic internal damage reduces the crucial fracture stress so that the bridging toughening value decreases.

The eutectic boundary is weak interface, thus when the eutectic is bridging the crack, the opening displacement will increase because of crack tip antedisplacement. There is a strong residual compressive stress in the eutectic matrix, thus the crack will extend along the eutectic grain boundary, resulting in the rod-shaped eutectic intergranular cracking and finishing the rod-shaped eutectic pull-out toughening mechanism. Energy will be consumed in the process of crack pull-out, thus the composites will be toughened.

The eutectic plastic deformation is very small, thus new fracture surface will be generated in the process of pull-out, and fracture work has something to do with fracture surface. Additional work of fracture will be calculated by accumulating points in the friction zone.

If $L_{0}$ is the original action length that friction has on the eutectic, and $0<L_{0}<L / 2$, then action area of the original friction is as follows.

$$
S=2 \pi R\left(L-L_{0}\right)
$$

If $\mathrm{x}$ is instantaneous pull-out length, then friction action length is calculated as $L^{\prime}=L_{0}+x$. Therefore, the work of friction in the sliding of the eutectic is as follows.

$$
W=\tau \int_{0}^{L_{0}}\left[2 \pi R\left(L-L_{0}-x\right)\right] d x
$$

If the boundary friction shear stress is $\tau=\mu \sigma_{n}, \mu$ is friction coefficient, and $\sigma_{n}$ is normal stress of the matrix in the eutectic along the interface. When the friction action length is $L_{0}$, the eutectic cross section area is $d S=\pi R^{2} / 4$, and the pull-out work in unit area is as follows.

$$
\Delta J_{2}=f_{f} \mu \sigma_{n} \frac{L_{0}\left(2 L-3 L_{0}\right)}{4 R}
$$

Suppose the average pull-out length of the eutectic is $L / 4$, then $L_{0}$ can be replaced by $L / 4$, the fracture work in the pull-out process is as follows.

$$
\Delta J_{2}=\frac{5 f_{f} \mu \sigma_{n} L^{2}}{64 R}
$$

It is shown in the type above that the eutectic volume content, interface friction coefficient and fibrous eutectic length have to be increased and the eutectic radius has to be decreased if a bigger pull-out fracture work value is a must.

According to energy dissipation, the relation of fracture toughness and energy dissipation can be described as $\Delta K_{C}=(E \Delta J)^{1 / 2}$. Suppose the eutectic aspect ratio is $\lambda=\frac{L}{2 R}$, then the eutectic pull-out 
toughening contribution to the material is as follows.

$$
\Delta K_{C 2}=\sqrt{\frac{5 f_{f} \mu \sigma_{n} E_{22}{ }^{\prime} \lambda L}{32}}
$$

Thus it can be seen that the eutectic pull-out toughening effect is connected with the interface shear strength that depends on the interface junction state. As for ceramic composite with damage eutectic and transformation particles, the junction of the eutectic and transformation particles is weak interface, thus it is convenient for the eutectic pull-out. Pull-out toughening is connected with damage eutectic elastic modulus, and the eutectic elastic modulus reduces when the eutectic is damaged, thus pull-out toughening effect is reduced by the eutectic internal damage.

According to synthesis type(15) and type(20), the crucial fracture stress and elastic modulus are decreased by the eutectic internal damage, thus bridging and pull-out toughening effect is reduced. However, apart from the rod-shaped eutectic bridging and pull-out toughening mechanism, zirconia particles surrounding the rod-shaped eutectic in the ceramic composite will generate transformation toughening mechanism because of stress induction.

\section{Conclusion}

Damage variable is introduced because of the rod-shaped eutectic microscopic structure. Loading function is defined according to maximum linear strain theory to analyze the rod-shaped eutectic rigidity attenuation characteristics and to determine the rod-shaped eutectic fracture stress when damage variable reaches the maximum.

Damage eutectic bridging toughening effect and pull-out toughening effect are analyzed according to the crack extension model of composite ceramic. It can be obtained from quantitative analysis that damage in the eutectic reduces the eutectic rigidity, thus fracture strength decreases and the eutectic contribution to composite ceramic bridging toughening and pull-out toughening decreases.

\section{References}

[1] Xinhua Ni, Zheng Jian, Xiequan Liu, Baohong Han. Strength Model of Eutectic Ceramic Composites [J]. Chinese Journal of Solid Mechanics, 2009, 30(2): 116-121.

[2] Xinhua Ni, Xiequan Liu, Baohong Han, Zhaogang Cheng, Guohui Zhong, Zhao Lei. Fracture Toughnes of Eutectic Ceramic Composite [J]. Chinese Journal of Theoretical and Applied Mechanics, 2010, 42(4): 682-690.

[3] Sayir A, Farmer S C. The Effect of the Microstructure on Mechanical Properties of Directionally Solidified $\mathrm{Al}_{2} \mathrm{O}_{3} / \mathrm{ZrO}_{2}\left(\mathrm{Y}_{2} \mathrm{O}_{3}\right)$ Eutectic[J]. Acta mater, 2000, 48: 4691-4697.

[4] L Lorca J, Orera V M. Directionally Solidified Eutectic Ceramic Oxides[J]. Progress in Materials Science, 2006, 51(6): 711-822.

[5] Zhongmin Zhao, Zhang Long, Hongbai Bai, Baocai Xu. Microstructures and Different-scale Multistage Toughening of Self-toughening Composite Ceramics with In-situ Growing Nano-micron Fibers[J]. Rare Metal Materials and Engineering, 2006, 35(S2): 91-95.

[6] Baofeng Li, Zheng Jian, Xinhua Ni, Yingchen Ma, Zhang Jing. Effective Elastic Constants of Fiber-eutectics and Transformation Particles Composite Ceramic[J], Advanced Materials Research, 2011, 177: 182-185.

[7] Xinhua Ni, Baofeng Li, Guohui Zhong, Zhao Lei. Size Dependent Thermal Expansion Coefficient of Rod-Shaped Oxide Eutectic Ceramic[J]. Advanced Material Research, 2010, 105-106: 146-149. 
[8] Sun Tao, Xinhua Ni, Xiequan Liu, Baohong Han, Zhaogang Cheng. Analysis of damage strain field for ceramic composite with eutectic interphases[J]. Chinese Journal of Computational Mechanics, 2012, 29: 527-531. 